\title{
Stereotactic body radiation therapy (SBRT) for the treatment of primary lung cancer in recipients of lung transplant
}

\author{
Assaf Moore ${ }^{1,3}$, Mordechai R. Kramer2,3, Dror Rosengarten ${ }^{2,3}$, Osnat Shtraichman²,3, \\ Alona Zer ${ }^{1,3}$, Elizabeth Dudnik ${ }^{1,3}$, Yasmin Korzets ${ }^{1}$, Aaron M. Allen ${ }^{1,3}$ \\ ${ }^{1}$ Institute of Oncology, Davidoff Cancer Center, Rabin Medical Center, Petach Tiqva, Israel \\ ${ }^{2}$ The Institute of Pulmonary Medicine, Rabin Medical Center, Petach Tiqva, Israel \\ ${ }^{3}$ Sackler Faculty of Medicine, Tel Aviv University, Tel Aviv, Israel
}

Radiol Oncol 2020; 54(2): 227-232.

Received 21 December 2019

Accepted 29 February 2020

Correspondence to: Aaron M. Allen, Radiotherapy Department, Davidoff Center, 39 Jabotinski St., Petach Tikvah, Israel. E-mail: ahron.alon@gmail.com

Disclosure: No potential conflicts of interest were disclosed.

Background. Lung transplantation is a life-saving treatment for patients with end stage lung disease. There may be a higher incidence of lung cancer in lung transplant recipients, and these cancers tend to be diagnosed at a more advanced stage. There is very little data on the safety and efficacy of stereotactic body radiation therapy (SBRT) for lesions in the native lung in lung-transplant recipients.

Patients and methods. A retrospective chart review of all patients who have undergone lung transplantation and were treated with SBRT for lung cancer in the native lung in the Davidoff Cancer Center was performed.

Results. Four patients who were treated with SBRT to a total of 5 lesions were included. Two patients were treated without histological confirmation of malignancy. All cases were discussed in a multidisciplinary tumor board before being referred for radiotherapy. Standard SBRT dosing was used. Responses were assessed by imaging. Three lesions exhibited a complete response and two lesions had a partial response. The patients who had partial responses developed distant metastases and died shortly. No patient developed measurable toxicity.

Conclusions. SBRT is effective and safe for the management of lung cancer in lung-transplant patients. Standard dose and fractionation can be used.

Key words: Stereotactic Body Radiation Therapy (SBRT); lung transplantation; lung cancer; radiotherapy

\section{Introduction}

Lung transplant is a life-saving last resort treatment for patients with end stage lung disease. The most common indications are chronic obstructive pulmonary disease (COPD) and idiopathic pulmonary fibrosis (IPF). ${ }^{1}$ This risk for lung cancer thus arises mainly from the native lung. ${ }^{1}$ There is a higher incidence of lung cancer in lung transplant recipients compared with the general population. ${ }^{2-6}$ This finding might be related to smoking, immunosuppression and/or underlying lung disease., ${ }^{4,5}$
Due to the improved life expectancy of transplanted patients and these predisposing factors, the incidence of lung cancer in lung transplant recipients is expected to increase. ${ }^{1}$ These cancers are often diagnosed in a more advanced stage and with a worse prognosis., ${ }^{3,4}$ For patients diagnosed with a potentially curable disease, curative treatment options include surgery and radiotherapy. We hereby present our experience with SBRT for the definitive treatment of four patients who underwent single lung transplantation and were diagnosed with lung cancer in the native lung. 


\section{Patients and methods}

\section{Patients}

The study includes all consecutive patients located in the registry of the Institute of Pulmonary Medicine who underwent lung transplantation and were treated with SBRT for lung cancer in the native lung at the Davidoff Cancer Center (DCC) at Rabin Medical Center (RMC) between June 2011 and June 2015.

\section{Data collection and outcomes}

This retrospective study was approved by the medical center's institutional Helsinki review board. No informed consent was required. Data were collected from medical records and included demographics, medical comorbidities, location and extent of disease, imaging findings, radiation treatment details, imaging and clinical follow-up, performance status, response to treatment, survival, and cause of death.

\section{Treatment planning}

Patients were immobilized for simulation using a customized vacuum cushion for CT simulation. Patients were simulated using a multiphase 4-dimentional CT simulation to monitor breathingrelated tumor motion. Images were reconstructed on the Advantage Workstation (GE Healthcare, Chicago, IL). An expansion in the cranio-caudal and axial dimensions for the internal target volumes (ITVs) was created based on tumor motion and location in 10 phases of breathing. The planning treatment volume (PTV) was defined as a 3-mm margin around the ITV. The PTV was reduced in case of proximity to vital normal tissue. Patients were treated with IMRT using dynamic sliding window multileaf collimator (MLC) or volumetric modulated arc therapy VMAT). Image guidance with cone beam CT preceded each fraction. Specification of the dose-volume histogram

TABLE 1. Dose-volume histogram constraints for organs at risk

\begin{tabular}{ll}
\hline Organ & Constraints \\
\hline Total Lung Dose (both lungs) & $\mathrm{V} 20<10 \%$ \\
Spinal Cord & Max dose $<18 \mathrm{~Gy}$ \\
Esophagus & Dose to $1 \mathrm{cc}<27 \mathrm{~Gy}$ \\
Heart & Dose to $1 \mathrm{cc}<30 \mathrm{~Gy}$ \\
\hline
\end{tabular}

$\mathrm{Gy}=\mathrm{Gray} ; \mathrm{V} 20=$ proportion of the lung receiving 20Gy
(DVH) constraints is available in Table 1. Dose calculations were performed using the Eclipse ${ }^{\mathrm{TM}}$ treatment planning system (Varian, Palo Alto, CA), AAA algorithm version 8. Treatment was prescribed to the $95 \%$ isodose line with PTV tolerance of $\pm 5 \%$. Quality assurance verification plans were performed with the ArcCHECK ${ }^{\mathrm{TM}}$ dosimeter (Sun Nuclear Corporation, Melbourne, FL). Before each treatment, image guided radiotherapy was used based on cone beam CT (CBCT) to position the patients.

\section{Post RT evaluation}

The treated tumors were assessed by CT or PET-CT eight weeks from completion of SBRT. Further imaging studies were scheduled at the treating physician's discretion.

\section{Results}

Four consecutive cases of lung transplant recipients who underwent SBRT for a lung lesion are included. All cases were discussed in a multidisciplinary team including pulmonologists, thoracic surgeons, radiation oncologists, medical oncologists and radiologists before being referred for radiotherapy. All patients were asymptomatic at diagnosis. Radiotherapy treatment parameters are summarized in Table 2.

\section{Case 1}

A 72-year-old man with a history of heavy smoking, COPD, hypertension, diabetes mellitus type 2, chronic renal failure, peripheral vascular disease and fatty liver disease. The patient underwent left lung transplant in 2006 due to severe emphysema, and was treated with tacrolimus and azathioprine to prevent rejection. In November 2011, an $8 \mathrm{~mm}$ nodule was detected in the right lower lobe. The lesion increased in size to $26 \mathrm{~mm}$ in May 2015. A biopsy yielded moderately differentiated squamous cell carcinoma. A positron emission tomographycomputed tomography (PET-CT) demonstrated high fluorodeoxyglucose (FDG) uptake in the nodule with no evidence of disease outside the lung. The patient was treated in June 2015 with a dose of 54 Gray (Gy) in three 18 Gy fractions. Treatment was well tolerated with no adverse events or measurable toxicity. Follow-up imaging demonstrated a complete response (CR). In February 2017, a new nodule appeared in the right lower lobe that in- 
TABLE 2. SBRT treatment parameters

\begin{tabular}{|c|c|c|c|c|c|}
\hline & $\begin{array}{c}\text { Case } 1 \\
1^{\text {st }} \text { course }\end{array}$ & $\begin{array}{c}\text { Case } 1 \\
2^{\text {nd }} \text { course }\end{array}$ & Case 2 & Case 3 & Case 4 \\
\hline Prescribed dose (Gy), number of fractions & $54 \mathrm{~Gy}, 3$ & 54 Gy, 3 & 60 Gy, 5 & $54 \mathrm{~Gy}, 3$ & $60 \mathrm{~Gy}, 5$ \\
\hline V5 - both lungs, (\%) & 15.6 & 21.5 & 18.9 & 10.2 & 27.1 \\
\hline V20 - both lungs, (\%) & 4.9 & 3.2 & 5.7 & 2.9 & 8 \\
\hline V20 - transplanted lung, (\%) & 7 & 0 & 2.3 & 0 & 0 \\
\hline
\end{tabular}

Gy $=$ Gray; MLD = mean lung dose, V5 = the \% of a structure's volume that receives 5 Gy; V20 = the \% of a structure's volume that receives 20 Gy

creased in size on follow-up imaging. A PET-CT in April 2017 demonstrated high FDG uptake in the nodule with no evidence of disease outside the lung. The patient was treated in July 2017 to a dose of $54 \mathrm{~Gy}$ in three $18 \mathrm{~Gy}$ fractions. The post SBRT PET-CT demonstrated shrinkage of the treated nodule, however, clear metastases in the liver and bone. A liver biopsy yielded adenocarcinoma of lung origin. The patient succumbed to metastatic disease in January 2018.

\section{Case 2}

A 76-year-old woman with a history of heavy smoking, COPD, diabetes mellitus type 2, chronic renal failure and atrial fibrillation. The patient underwent left lung transplant in 2002 due to severe emphysema, and was treated with tacrolimus and mycophenolic acid to prevent rejection. In June 2010, a central $0.5 \mathrm{~mm}$ nodule was detected in the right lower lobe. The lesion increased in size up to $13 \mathrm{~mm}$ in October 2011, and demonstrated high FDG uptake with no evidence of disease outside the lung. The patient was discussed in a multidisciplinary tumor board and it was decided to treat the lesion with SBRT without histological confirmation. The patient was treated in February 2012 to a dose of 60 Gy in five 12 Gy fractions. Treatment was well tolerated with no adverse events or measurable toxicity. Follow-up imaging demonstrated a complete response (Figure 1). The patient passed away in May 2018 secondary to pneumonia and sepsis.

\section{Case 3}

A 72-year-old man with a history of hypertension, diabetes mellitus type 2 , chronic renal failure and ischemic heart disease. Underwent right lung transplant in 2002 due to IPF, and was treated with tacrolimus and mycophenolic acid to prevent rejection. In 2013 a nodule was detected in the left lower lobe and increased in size to $15 \mathrm{~mm}$ in October 2013. There was no FDG uptake in the nodule. The patient was discussed in a multidisciplinary tumor board and it was decided to treat the lesion

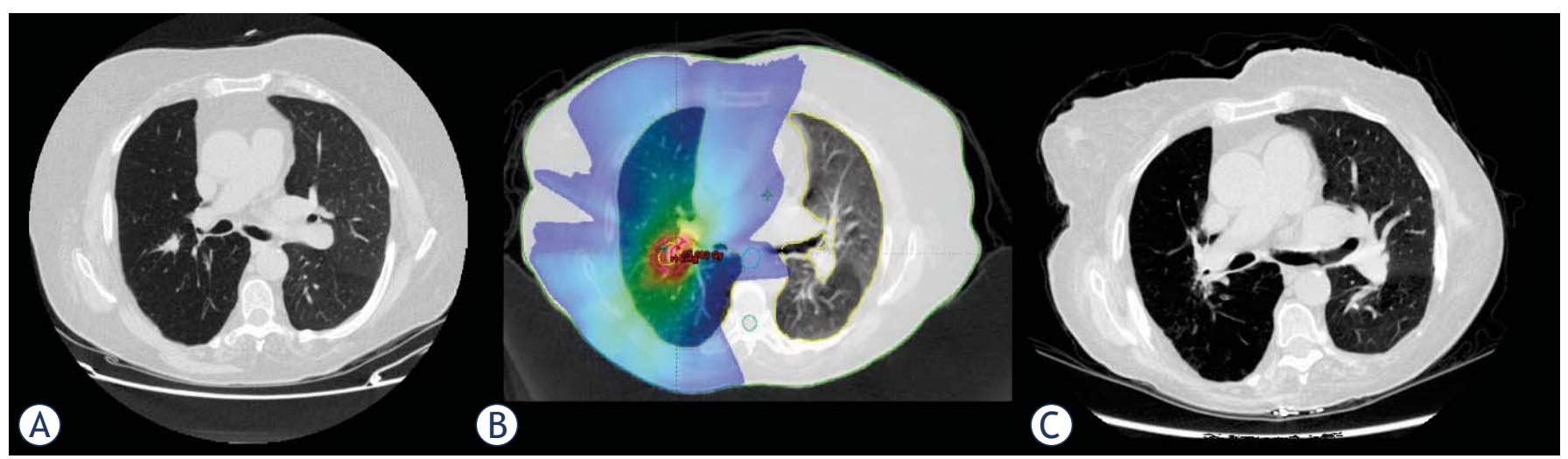

FIGURE 1. (A) Pretreatment CT demonstrating a central nodule in the right lower lobe (B) Radiation field arrangement and dose color wash for SBRT (C) CT 2 months after treatment completion demonstrating a complete disappearance of the target nodule. 


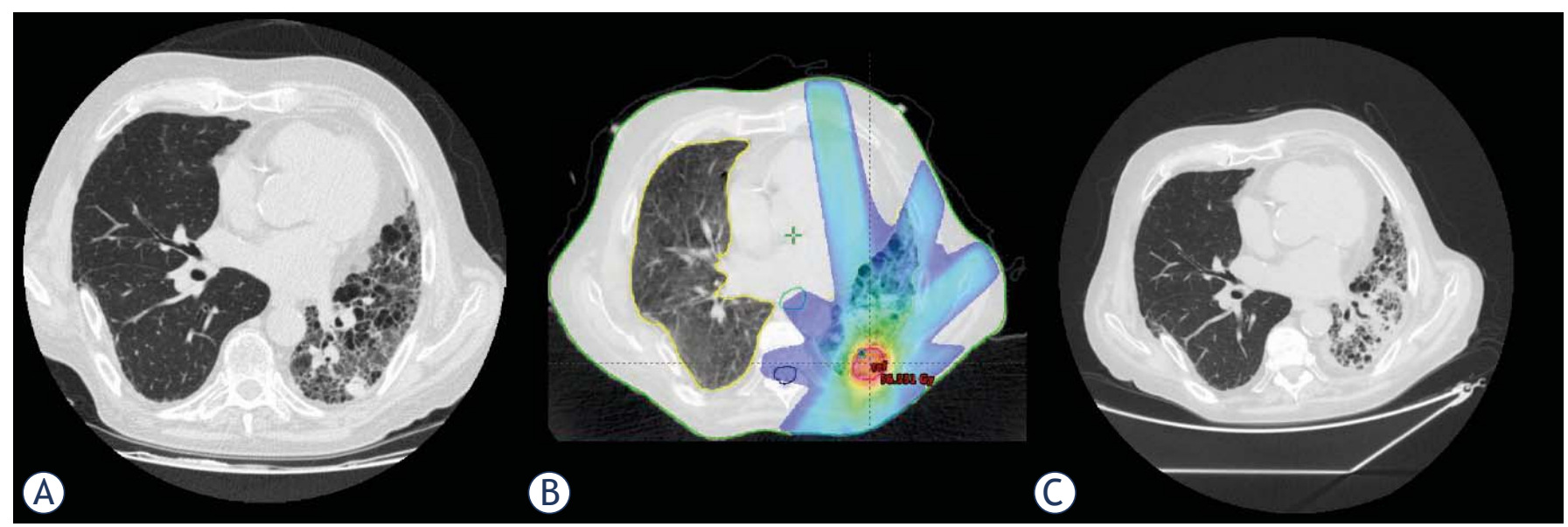

FIGURE 2. (A) Pretreatment CT demonstrating a nodule was detected in the left lower lobe (B) Radiation field arrangement and dose color wash for SBRT (C) CT 2 months after treatment completion demonstrating a complete disappearance of the target nodule.

with SBRT without histological confirmation. The patient was treated in February 2012 to a dose of 54 Gy in three 18 Gy fractions. Treatment was well tolerated with no adverse events or measurable toxicity. The post SBRT, CT scans yielded a disappearance of the treated nodule (Figure 2). The patient passed away in October 2015 secondary to bacteremia and sepsis.

\section{Case 4}

A 65-year-old man with a history of hypertension and diabetes mellitus type 2 . Underwent left lung transplant in 2009 due to severe IPF, and was treated with tacrolimus and mycophenolic acid to prevent rejection. In 2011, a mass was detected in the anterior right mediastinum with involvement of the pleura and right upper lobe. A PET-CT demonstrated high FDG uptake in the mass that increased in size to $3.8 \mathrm{~cm}$ with no evidence of disease outside the lung. A biopsy yielded moderately differentiated small cell lung cancer (SCLC). The patient was discussed in a multidisciplinary tumor board, and determined to be a poor surgical candidate, was referred for SBRT and then planned for "adjuvant" chemotherapy. The patient was treated in June 2011 to a dose of 60 Gy in five 12 Gy fractions. Treatment was well tolerated with no adverse events or measurable toxicity. The post SBRT, CT scans yielded a partial response (PR) of the treated nodule (Figure 3), however, appearance of metastases in the liver and nodes above and below the diaphragm. The patient succumbed to a rapid progression of metastatic disease in August 2011.

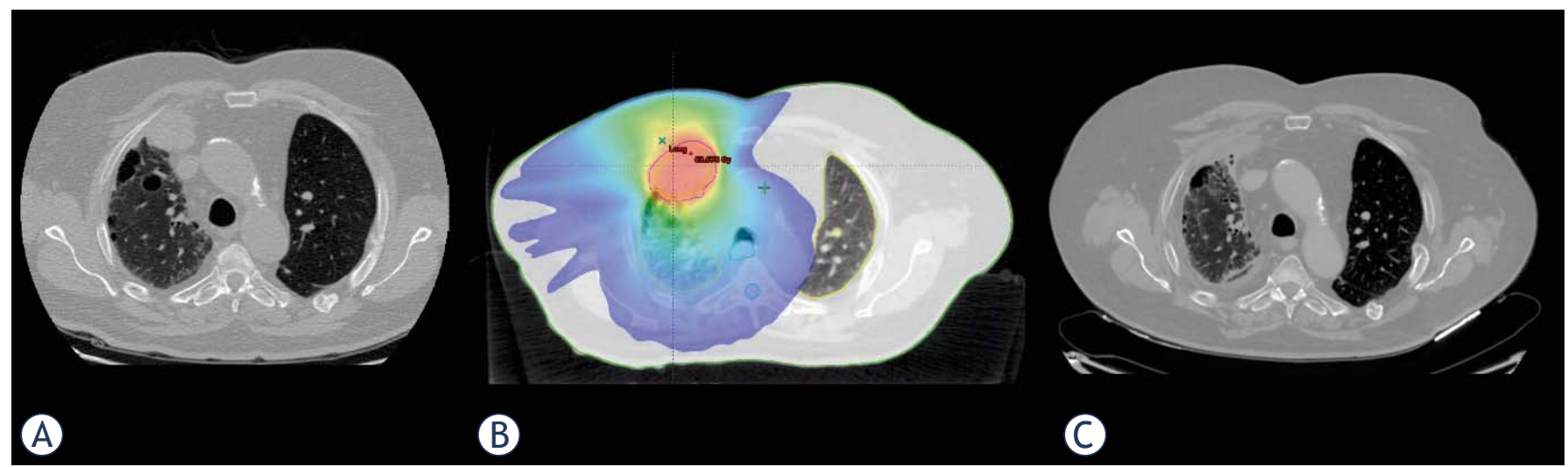

FIGURE 3. (A) Pretreatment CT demonstrating a mass in the anterior right mediastinum with involvement of the pleura and right upper lobe (B) Radiation field arrangement and dose color wash for SBRT (C) CT 2 months after treatment completion demonstrating regression of the target nodule. 


\section{Discussion}

Lung cancer in lung transplant recipients is a unique clinical scenario, in which patients suffer from at least two major life-threatening conditions. Treatment options for these patients may be limited due to the underlying condition, other comorbidities and their immunosuppressed state. Treating these patients requires a multi-disciplinary effort. Characterizing this group of patients is beyond the scope of this report.

We present a series of 4 consecutive patients who were treated with SBRT to a total of 5 lesions in the native lungs. One patient had proven squamous cell carcinoma and then adenocarcinoma, one had small cell carcinoma, and two were treated without histological confirmation of malignancy.

Surgery remains the standard-of-care treatment for medically-operable early stage non-small cell lung cancer. Radiotherapy for the definitive treatment of early lung cancer was traditionally indicated for the medically-inoperable or those refusing surgery. ${ }^{7}$ Lung resection is challenging in patients with a major pulmonary disease, and especially when these patients have undergone lung transplantation and are immunosuppressed. Specific concerns include Impaired wound healing and anastomotic complications. ${ }^{8}$ Data on the safety of these procedures is scarce. In one series that included both malignant and benign/infectious causes for pulmonary resection of the native or allograft, of the 11 patients included, 3 patients died of post-procedural infectious complications, 2 died of acute respiratory distress syndrome and organ failure and 1 died as a result of bronchiolitis obliterans organizing pneumonia. ${ }^{8}$

Radiotherapy is an integral modality in the treatment of early lung cancer. SBRT has been studied extensively in medically inoperable patients, and may in fact achieve better control than standard radiotherapy. ${ }^{7,9,10}$ SBRT has also been proven effective for medically operable patients, even compared with surgery. ${ }^{11,12}$ While it is unknown whether radiotherapy and SBRT specifically can be safely and effectively used in lung transplant recipients, toxicity in clinical trials has been low with no treatment-related mortality. ${ }^{7,9,10}$ Data on the interaction between immunosuppression and advanced oncologic treatments is scarce. This applies to systemic therapy such as tyrosine kinase inhibitors, but even more concerning - with immunotherapeutic agents facing a risk for organ rejection. ${ }^{13}$ The immune system's role in mediating response to radiotherapy is being studied, however the exact implica- tions of immunosuppression during radiotherapy are poorly understood..$^{14}$ In this series, all lesions achieved excellent response by imaging -3 lesions exhibited a complete response and two lesions partial responses. The patients who had partial responses developed distant metastases and died shortly, thus; the maximal response may not have been achieved during the short follow-up. None of the lesions who exhibited a complete response recurred locally. Based on this small series, even in immunosuppressed patients, SBRT to lung lesions is effective at achieving local control.

There have been several reports of SBRT in the treatment of patients with lung cancer who had previously undergone solid organ transplant. In a series of 15 patients ( 9 of which underwent lung transplant), no patients experienced grade 3 or 4 toxicity, however, one patient with a history of single lung transplant died of radiation pneumonitis 7 months after SBRT to a lesion within the transplanted lung. The cumulative incidence of local failure was $7 \%$ and $13 \%$ at 1 and 2 year and the incidence of distant failure was $40 \%$ at 1 year. ${ }^{15}$ In another series, two patients were treated with SBRTrange dosing sequentially with chemotherapy. One achieved a durable complete response, while another progressed shortly. ${ }^{4}$ One case ineligible for surgical management of stage IB adenocarcinoma arising from a donor lung post-double lung transplantation, was safely and effectively treated to a dose of $60 \mathrm{~Gy}$ in 8 fractions. ${ }^{16}$

The doses and organs at risk constraints we have used did not differ from our institutional policy towards non-transplanted patients and seemed to be effective and safe. However, whether an optimal dose and fractionation exists or whether tighter constraints are required, has not been determined and no clear recommendation can be made.

While there were no cases of grade 2 or higher pneumonitis in our patients, organ transplanted patients, and specifically lung transplanted patients could prove a diagnostic challenge for this type of toxicity, as the differential diagnosis for clinical deterioration is broad and might not be distinguishable by imaging.

The main limitations of this study relate to its retrospective design and lack of a control arm, which are generally associated with methodological biases and difficulties in results interpretation. The most concerning bias in our study is clearly associated with patient selection, as patients who have undergone transplantation usually have other comorbidities and their cancers may have a more aggressive course. 
It is clear that SBRT is a safe and efficient modality for the treatment of recipients of lung transplant and could offer long-term control and potentially a definitive solution. There are no randomized comparative reports comparing it with invasive procedures. Physicians must consider the potential early and late toxicities of thoracic radiation, including pneumonitis, esophagitis and vascular toxicity. These may be avoided by minimizing volumes and strictly maintaining dose constraints. SBRT could serve to achieve these objectives.

\section{Conclusions}

SBRT for the management of lung cancer in the native lung in lung-transplanted patients is effective and safe and can offer long-term control. Standard dose and fractionation can be used. It should be considered in clinical situations where surgical procedures are not feasible. These results should encourage clinicians to investigate its role further, potentially in place of invasive procedures.

\section{References}

1. Mathew J, Kratzke RA. Lung cancer and lung transplantation: a review. Thorac Oncol 2009; 4: 753-60. doi: 10.1097/JTO.0b013e31819afdd9

2. Choi $\mathrm{YH}$, Leung AN, Miro S, Poirier $\mathrm{C}$, Hunt $\mathrm{S}$, Theodore J. Primary bronchogenic carcinoma after heart or lung transplantation: radiologic and clinical findings. J Thorac Imaging 2000; 15: 36-40. doi: 10.1097/00005382 200001000-00008

3. Raviv $Y$, Shitrit D, Amital A, Fox B, Rosengarten D, Fruchter O, et al. Lung cancer in lung transplant recipients: experience of a tertiary hospital and literature review. Lung Cancer 2011; 74: 280-3. doi: 10.1016/j.lungcan.2011.02.012

4. Du L, Pennell NA, Elson P, Hashemi-Sadraei N. Lung cancer treatment outcomes in recipients of lung transplant. Transl Lung Cancer Res 2015; 4: 784-91. doi: 10.3978/j.issn.2218-6751.2015.12.08

5. Collins J, Kazerooni EA, Lacomis J, McAdams HP, Leung AN, Shiau M, et al. Bronchogenic carcinoma after lung transplantation: frequency, clinical characteristics, and imaging findings. Radiology 2002; 224: 131-8. doi: 10.1148/ radiol.2241011189

6. Dickson RP, Davis RD, Rea JB, Palmer SM. High frequency of bronchogenic carcinoma after single-lung transplantation. J Heart Lung Transplant 2006; 25: 1297-301. doi: 10.1016/j.healun.2006.09.009

7. Timmerman RD, Hu C, Michalski J, Straube W, Galvin J, Johnstone D, et al. Long-term results of RTOG 0236: a phase II trial of stereotactic body radiation therapy (SBRT) in the treatment of patients with medically inoperable stage I non-small cell lung cancer. Int J Radiat Oncol Biol Phys 2014; 90: S30. doi: 10.1016/j.jijrobp.2014.05.135

8. Fitton TP, Bethea BT, Borja MC, Yuh DD, Yang SC, Orens JB, et al. Pulmonary resection following lung transplantation. Ann Thorac Surg 2003; 76: 1680-6. doi: 10.1016/s0003-4975(03)00975-5

9. Nyman J, Hallqvist A, Lund J-A, Brustugun OT, Bergman B, Bergström P, et al. SPACE - A randomized study of SBRT vs conventional fractionated radiotherapy in medically inoperable stage I NSCLC. Radiother Oncol 2016; 121 1-8. doi: 10.1016/j.radonc.2016.08.015
10. Ball D, Mai GT, Vinod S, Babington S4, Ruben J5, Kron T, et al. Stereotactic ablative radiotherapy versus standard radiotherapy in stage 1 non-smallcell lung cancer (TROG 09.02 CHISEL): a phase 3, open-label, randomised controlled trial. Lancet Oncol 2019; 20: 494-503. doi: 10.1016/S14702045(18)30896-9

11. Chang JY, Senan S, Paul MA, Mehran RJ, Louie AV, Balter P, et al. Stereotactic ablative radiotherapy versus lobectomy for operable stage I non-small-cell lung cancer: a pooled analysis of two randomised trials. Lancet Oncol 2015; 16: 630-7. doi: 10.1016/S1470-2045(15)70168-3

12. Zheng X, Schipper M, Kidwell K, Lin J, Reddy R, Ren Y, et al. Survival outcome after stereotactic body radiation therapy and surgery for stage I non-small cell lung cancer: a meta-analysis. Int J Radiat Oncol Biol Phys 2014; 90: 60311. doi: 10.1016/j.jirobp.2014.05.055

13. Ros J, Matos I, Martin-Liberal J. Immunotherapy in organ-transplanted cancer patients: efficacy and risk of organ rejection. Ann Oncol 2019; 30: 1173-7. doi: 10.1093/annonc/mdz129

14. Walle T, Martinez Monge R, Cerwenka A, Ajona D, Melero I, Lecanda F. Radiation effects on antitumor immune responses: current perspectives and challenges. Ther Adv Med Oncol 2018; 10: 1758834017742575 . doi: $10.1177 / 1758834017742575$

15. Fleming CW, Stephans KL, Broughman JR, Rybicki L, Budev M, Ahmad U, et al. Outcomes after SBRT for inoperable early stage lung cancers arising in organ transplant patients. Int J Radiat Oncol Biol Phys 2019; 105: E486. doi: 10.1016/j.jirobp.2019.06.1382

16. Chen H, Tikkanen J, Boldt RG, Louie A V. Stereotactic ablative radiotherapy for early-stage lung cancer following double lung transplantation. Radiat Oncol 2018; 13: 142. doi: 10.1186/s13014-018-1089-8 